INP-98-7/508, MPI/PhT/98-19, hep-ph/9802429, February 1998

\title{
Three-loop vacuum integrals in FORM and REDUCE
}

\author{
P. A. BAIKOV' \\ Institute of Nuclear Physics, Moscow State University, \\ 119 899, Moscow, Russia \\ M. STEINHAUSER \\ Max-Planck-Institut für Physik, Werner-Heisenberg-Institut, \\ D-80805 Munich, Germany
}

\begin{abstract}
The implementation of an algorithm for three-loop massive vacuum integrals, based on the explicit solution of the recurrence relations, in REDUCE and FORM is described.
\end{abstract}

\section{Introduction}

The increasing experimental precision makes it mandatory to compute higher order corrections to physical quantities. This essentially requires the evaluation of multi-loop integrals. However, even at two loops it is very often not possible to solve the integrals exactly and one has to rely on approximations. A very powerful approach to get, nevertheless, reasonable results is based on expansions in small quantities. Recently a method was developed for three-loop polarization functions combining expansions from different kinematical regions with the help of conformal mapping and Padé approximation [1,2] . An essential ingredient for this procedure are massive integrals with vanishing external momentum. For their computation usually recurrence relations are used which are based on the integration-by-parts technique [3,4]. These relations connect Feynman integrals with different powers of their denominators. In many cases they provide a possibility to express an integral with given degrees of the denominators as a linear combination of a few so-called master integrals with prefactors which are rational functions of the spacetime dimension $D$. The construction of such a procedure is a nontrivial problem even at two-loop level [5]. At three loops up to now only the case of vacuum integrals with one

$\overline{1}$ Supported in part by INTAS (grant 93-0744-ext), Volkswagen Foundation (contract No. I/73611)

Email: baikov@theory.npi.msu.su 
non-zero mass and various numbers of massless lines has been considered [4,6]. For the problems of practical interest the direct application of these equations usually leads to intermediate expressions which need several hundred megabyte up to a few gigabyte of disk space.

For the two-loop massless master integral some years ago the recurrence procedure has been solved explicitly [7]. The solution, expressed in terms of multiple sums, is also used in practice [8] - at least for those cases where the exponents of the propagators are not too small. Concerning the general multi-loop case in [9] a new approach to implement recurrence relations [3] was suggested. There the factors in front of the master integrals, which in the following are called coefficient functions, are considered as independent solutions of the recurrence relations. In [9] integral representations for these solutions were obtained. It appeared, that in some cases these representations can be expressed in terms of Pochhammer symbols. As an example the vacuum integrals with four equal masses and two massless lines has been considered, and the efficiency of this approach was demonstrated by the calculation of the three-loop QED vacuum polarization.

In this paper we describe the algorithm, suggested in [9], in more details and discuss some peculiarities of its implementation in REDUCE [10] and FORM [11]. In Section 2 the general $L$-loop case is considered. In Section 3 the derived formulas are specified to three loops and explicit solutions are given. The implementation is discussed in Section 4 and finally Section 5 contains our conclusions.

\section{Basic formulas}

Let us in a first step derive the recurrence relations. The combinatoric structure of these relations becomes more transparent if we start with the general multi-loop case, keeping in mind, however, the application at three-loop level. Therefore let us consider $L$-loop vacuum integrals with $N=L(L+1) / 2$ denominators (This number of denominators provides the possibility to express any scalar product of loop momenta as linear combination of the denominators; the diagrams of practical interest which usually have less number of denominators, can be considered as special cases with some exponents equal to zero.):

$$
B(\underline{n}, D) \equiv B\left(n_{1}, \ldots, n_{N}, D\right)=\frac{m^{2 \Sigma n_{i}-L D}}{\left[\imath \pi^{D / 2} \Gamma(3-D / 2)\right]^{L}} \int \cdots \int \frac{d^{D} p_{1} \ldots d^{D} p_{L}}{D_{1}^{n_{1}} \ldots D_{N}^{n_{N}}}
$$

where $p_{i}(i=1, \ldots, L)$ are loop momenta and $D_{a}=A_{a}^{i j} p_{i} \cdot p_{j}-\mu_{a} m^{2}(\mathrm{a}=1, \ldots, \mathrm{N} ;$ here and below the sum over repeated indices is understood). For convenience we set $m=1$ in the following. The recurrence relations are obtained by acting with $\left(\partial / \partial p_{i}\right) \cdot p_{k}$ on the integrand [3]: 


$$
D \delta_{k}^{i} B(\underline{n}, D)=2 \tilde{A}_{k l}^{a}\left(\mathbf{I}_{a}^{-}+\mu_{a}\right) A_{d}^{i l} \mathbf{I}^{d+} B(\underline{n}, D)
$$

where $\mathbf{I}_{c}^{-} B\left(\ldots, n_{c}, \ldots\right) \equiv B\left(\ldots, n_{c}-1, \ldots\right)$ and $\mathbf{I}_{c}^{+} B\left(\ldots, n_{c}, \ldots\right) \equiv n_{c} B\left(\ldots, n_{c}+1, \ldots\right)$ (here, no sum over $n_{c}$ may be performed). The factors $\tilde{A}_{k l}^{a}$ arise from the fact that the scalar products $p_{i} \cdot p_{j}$ in the numerator which appear as a result of the differentiation are expressed in terms of the denominators $D_{a}$ via:

$$
p_{k} \cdot p_{l}=\tilde{A}_{k l}^{a}\left(D_{a}+\mu_{a}\right)
$$

Using the identities

$$
\left[\mathbf{I}_{a}^{-}, \mathbf{I}^{d+}\right]=\delta_{a}^{d}, \quad A_{a}^{i l} \tilde{A}_{k j}^{a}=\frac{1}{2}\left(\delta_{k}^{i} \delta_{j}^{l}+\delta_{j}^{i} \delta_{k}^{l}\right)
$$

the recurrence relations (2) can be represented as

$$
(D-L-1) \delta_{k}^{i} B(\underline{n}, D)=2 A_{d}^{i l} \mathbf{I}^{d+} \tilde{A}_{k l}^{a}\left(\mathbf{I}_{a}^{-}+\mu_{a}\right) B(\underline{n}, D),
$$

where we have exploited that the matrices $A^{a}$ and $\tilde{A}^{a}$ can be chosen to be symmetrical. If we denote

$$
\tilde{A}_{k l}^{a}\left(\mathbf{I}_{a}^{-}+\mu_{a}\right) \equiv \mathbf{A}_{k l}, \quad A_{d}^{i l} \mathbf{I}^{d+} \equiv \partial^{i l},
$$

Eq. (3) will read:

$$
\left[\boldsymbol{\partial}^{i l} \cdot \mathbf{A}_{k l}-\frac{D-L-1}{2} \delta_{k}^{i}\right] B(\underline{n}, D)=0 \text {. }
$$

Let us now diagonalize these relations with respect to the operators $\boldsymbol{\partial}^{i l}$. Therefore we multiply Eq. (4) with $\partial^{j k} \operatorname{det}(\mathbf{A})$, which is the cofactor of the matrix element $\mathbf{A}_{k l}$, and sum afterwards over $k$. Finally we arrive at:

$$
\left[\partial^{i l} \cdot \operatorname{det}(\mathbf{A})-\frac{D-L-1}{2}\left(\partial^{i l} \operatorname{det}(\mathbf{A})\right)\right] B(\underline{n}, D)=0 .
$$

Our goal is to find the coefficient functions $f^{k}(\underline{n}, D)$ which relate the given integral $B(\underline{n}, D)$ with the basic master integrals $B\left(\underline{n}_{k}, D\right)$ :

$$
B(\underline{n}, D)=f^{k}(\underline{n}, D) B\left(\underline{n}_{k}, D\right),
$$


where the coefficient functions have to fulfill the initial conditions $f^{i}\left(\underline{n}_{k}, D\right)=\delta_{k}^{i}$. The index $k$ is used to label different sets of indices $\underline{n}$ which are fixed by the choice of the master integrals. Assuming that the master integrals are algebraically independent one can conclude that the functions $f^{k}(\underline{n}, D)$ should be independent solutions of the recurrence relations. This means that as soon as we find a set of independent solutions it is possible to construct the desired coefficient functions as linear combinations respecting the given initial conditions. To this end it turns out that it is useful to construct an auxiliary integral representation for these functions where the operators can be written in the form $2: \mathbf{I}^{d+} \rightarrow \partial / \partial x_{d}, \mathbf{I}_{d}^{-} \rightarrow x_{d}$. Then the differential equation corresponding to Eq. (3) has the solution $g\left(x_{a}\right)=\operatorname{det}(\mathbf{A})^{(D-L-1) / 2}=P\left(x_{a}+\mu_{a}\right)^{(D-L-1) / 2}$, where $P\left(x_{a}\right)$ is a polynomial in $x_{a}$ of degree $L$ :

$$
P\left(x_{a}\right)=\operatorname{det}\left(\tilde{A}_{k l}^{a} x_{a}\right) .
$$

Therefore it is tempting to consider the "Laurent" coefficients of the function $g\left(x_{a}\right)$ in order to determine the coefficient functions:

$$
f^{k}(\underline{n}, D)=\frac{1}{(2 \pi \imath)^{N}} \oint \cdots \oint \frac{d x_{1} \cdots d x_{N}}{x_{1}^{n_{1} \cdots x_{N}}} \operatorname{det}\left(\tilde{A}_{i l}^{a}\left(x_{a}+\mu_{a}\right)\right)^{(D-L-1) / 2} .
$$

Here the integral symbols denote $N$ subsequent complex integrations. The contours depend on the index $k$ and will be specified below. Acting with Eq. (3) on (5) leads (up to surface terms) to the corresponding differential operator acting on $g\left(x_{a}\right)$, which gives zero. The surface terms can be removed by a proper choice of the complex contours: either closed contours or integration paths which end in infinity. For the last case one has to consider analytical continuations of $D$ from large negative values. Note that Eq. (5) is a solution of relation (2) and thus the different choices of the contours correspond to different solutions which are enumerated with the index $k$.

The solutions (㺃) satisfy by construction the following condition:

$$
f^{k}(\underline{n}, D)=P\left(\mathbf{I}_{a}^{-}+\mu_{a}\right) f^{k}(\underline{n}, D-2) .
$$

In general, however, we are interested in coefficient functions, $\tilde{f}^{k}(\underline{n}, D)$, which correspond to a specific set of master integrals and hence a linear combination of $f^{k}(\underline{n}, D)$ with coefficients depending on $D$ has to be considered. Then Eq. (6) gets more complicated as a mixing among the different functions is possible:

$$
\tilde{f}^{k}(\underline{n}, D)=S_{i}^{k}(D) P\left(\mathbf{I}_{a}^{-}+\mu_{a}\right) \tilde{f}^{i}(\underline{n}, D-2),
$$

$\overline{2}$ Note that before the transition to this representation is performed the order of the operators has to be reversed. 
where $S_{i}^{k}(D)$ are rational functions of the space-time dimension $D$ only.

\section{Three-loop case}

In this section we specify the general $L$-loop formulas derived in the previous one to the three-loop case with four equal masses and two massless lines, i.e., $\mu_{1}=\mu_{2}=0, \mu_{3}=$ $\mu_{4}=\mu_{5}=\mu_{6}=1$. Then Eq. (1) becomes:

$$
B(\underline{n}, D)=\frac{m^{2 \Sigma_{1}^{6} n_{i}-3 D}}{\left[\imath \pi^{D / 2} \Gamma(3-D / 2)\right]^{3}} \iiint \frac{d^{D} p d^{D} k d^{D} l}{D_{1}^{n_{1}} D_{2}^{n_{2}} D_{3}^{n_{3}} D_{4}^{n_{4}} D_{5}^{n_{5}} D_{6}^{n_{6}}},
$$

with

$$
\begin{aligned}
& D_{1}=k^{2}, D_{2}=l^{2}, D_{3}=(p+k)^{2}-m^{2}, D_{4}=(p+l)^{2}-m^{2}, \\
& D_{5}=(p+k+l)^{2}-m^{2}, D_{6}=p^{2}-m^{2} .
\end{aligned}
$$

The coefficient functions from Eq. (5) read:

$$
f(\underline{n}, D)=\frac{1}{(2 \pi \imath)^{6}} \oint \frac{d x_{1}}{x_{1}^{n_{1}}} \cdots \oint \frac{d x_{6}}{x_{6}^{n_{6}}} P\left(x_{1}, x_{2}, x_{3}+1, \ldots, x_{6}+1\right)^{D / 2-2}
$$

where the polynomial $P\left(x_{1}, \ldots, x_{6}\right)$ is given by

$$
\begin{aligned}
P\left(x_{1}, \ldots, x_{6}\right)= & \left(x_{1}+x_{2}\right)\left(x_{1} x_{2}-x_{3} x_{4}-x_{5} x_{6}\right)+\left(x_{3}+x_{4}\right)\left(-x_{1} x_{2}+x_{3} x_{4}-x_{5} x_{6}\right) \\
& +\left(x_{5}+x_{6}\right)\left(-x_{1} x_{2}-x_{3} x_{4}+x_{5} x_{6}\right)+x_{1} x_{3} x_{6}+x_{1} x_{4} x_{5}+x_{2} x_{3} x_{5} \\
& +x_{2} x_{4} x_{6} .
\end{aligned}
$$

In this equation we have omitted an overall factor $(-1 / 4)$ as it would lead to a trivial rescaling which cancels after considering initial conditions.

Let us now compute the coefficient functions which correspond to the choice of the master integrals given in [4, 6] where $B(\underline{n}, D)$ has been written in the form:

$$
\begin{aligned}
B(\underline{n}, D)= & N(\underline{n}, D) B(0,0,1,1,1,1, D)+M(\underline{n}, D) B(1,1,0,0,1,1, D) \\
& +T(\underline{n}, D) B(0,0,0,1,1,1, D) .
\end{aligned}
$$

This leads us to the following normalization conditions:

$$
N(0,0,1,1,1,1, D)=1, \quad N(1,1,0,0,1,1, D)=0, \quad N(0,0,0,1,1,1, D)=0,
$$




$$
\begin{array}{ccc}
M(0,0,1,1,1,1, D)=0, & M(1,1,0,0,1,1, D)=1, & M(0,0,0,1,1,1, D)=0, \\
T(0,0,1,1,1,1, D)=0, & T(1,1,0,0,1,1, D)=0, & T(0,0,0,1,1,1, D)=1 .
\end{array}
$$

In a first step the function $N(\underline{n}, D)$ is considered. The last two conditions of Eq. (8) are satisfied if the contours for the massive indices are chosen to be small circles around zero, i.e., $x_{i}=0$. In practice it is useful to perform a Taylor expansion in $x_{3}, x_{4}, x_{5}$ and $x_{6}$ where, according to the residuum theorem, only one term leads to a result different from zero (Here and in the following the proportional sign ( $\propto$ ") is used as the $D$ dependent factor is fixed at the end in accordance with the normalization given in Eq. (8).):

$$
\left.N(\underline{n}, D) \propto \oint \oint \frac{d x_{1} d x_{2}}{x_{1}^{n_{1}} x_{2}^{n_{2}}}\left[\frac{\partial_{3}^{n_{3}-1} \ldots \partial_{6}^{n_{6}-1}}{\left(n_{3}-1\right) ! \ldots\left(n_{6}-1\right) !} P\left(x_{1}, x_{2}, x_{3}+1, \ldots, x_{6}+1\right)^{D / 2-2}\right]\right|_{x_{3}, \ldots, x_{6}=0} .
$$

The remaining integrals over $x_{1}$ and $x_{2}$, for which in general (because of the derivatives) the exponent of the polynomial $P\left(x_{1}, \ldots, x_{6}\right)$ is reduced, can easily be expressed in terms of Pochhammer symbols $(a)_{n}=\Gamma(a+n) / \Gamma(a)$ :

$$
\oint \oint \frac{d x_{1} d x_{2}}{x_{1}^{n_{1}} x_{2}^{n_{2}}} P\left(x_{1}, x_{2}, 1,1,1,1\right)^{D / 2-2-c} \propto \frac{(D / 2-1)_{-c}(4-3 D / 2)_{n_{1}+n_{2}+3 c}}{4^{\left(n_{1}+n_{2}+3 c\right)}(2-D / 2)_{n_{1}+c}(2-D / 2)_{n_{2}+c}},
$$

where $\Gamma(x)$ is Eulers $\Gamma$ function.

Let us next turn to the function $M(\underline{n}, D)$ which can be treated in analogy. The only difference is that due to the symmetry $B(1,1,0,0,1,1, D)=B(1,1,1,1,0,0, D)$ it is profitable to consider the sum of the solutions with a Taylor expansion performed in the variables $\left(x_{1}, x_{2}, x_{3}, x_{4}\right)$ and $\left(x_{1}, x_{2}, x_{5}, x_{6}\right)$ :

$$
M(\underline{n}, D)=m\left(n_{1}, n_{2}, n_{3}, n_{4}, n_{5}, n_{6}, D\right)+m\left(n_{1}, n_{2}, n_{5}, n_{6}, n_{3}, n_{4}, D\right),
$$

where $m(\underline{n}, D)$ is given by

$$
\left.m(\underline{n}, D) \propto \oint \oint \frac{d x_{5} d x_{6}}{x_{5}^{n_{5}} x_{6}^{n_{6}}}\left[\frac{\partial_{1}^{n_{1}-1} \ldots \partial_{4}^{n_{4}-1}}{\left(n_{1}-1\right) ! \ldots\left(n_{4}-1\right) !} P\left(x_{1}, x_{2}, x_{3}+1, \ldots, x_{6}+1\right)^{D / 2-2}\right]\right|_{x_{1}, \ldots, x_{4}=0} .
$$

Again the remaining integrals are easily performed with the result

$$
\begin{aligned}
& \oint \oint \frac{d x_{5} d x_{6}}{x_{5}^{n_{5}} x_{6}^{n_{6}}} P\left(0,0,1,1, x_{5}+1, x_{6}+1\right)^{D / 2-2-c}
\end{aligned}
$$

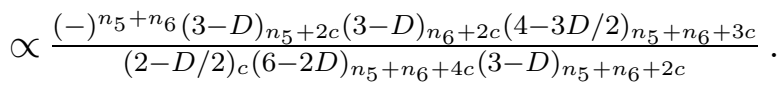

The case " $\mathrm{T}$ " is more complicated. The conditions (9) show that we should find a solution 
which is non-zero if one of the massive indices is non-positive. It turns out that the following combination of "Taylor" solutions leads to the desired result:

$$
\begin{aligned}
T\left(n_{1}, n_{2}, n_{3}, n_{4}, n_{5}, n_{6}, D\right)= & t\left(n_{1}, n_{2}, n_{3}, n_{4}, n_{5}, n_{6}, D\right)+t\left(n_{1}, n_{2}, n_{4}, n_{3}, n_{6}, n_{5}, D\right) \\
& +t\left(n_{1}, n_{2}, n_{5}, n_{6}, n_{3}, n_{4}, D\right)+t\left(n_{1}, n_{2}, n_{6}, n_{5}, n_{4}, n_{3}, D\right),
\end{aligned}
$$

where

$$
t(\underline{n}, D)=0 \quad \text { if } \quad n_{4} \text { or } n_{5} \text { or } n_{6}<1 .
$$

Four terms are necessary in order to meet the symmetry properties of the initial integral. From Eq. (7) the following general representation for the solution with the property given in Eq. (10) is obtained:

$$
\begin{aligned}
\bar{t}(\underline{n}, D) \propto \oint & \oint \frac{d x_{1} d x_{2} d x_{3}}{x_{1}^{n_{1}} x_{2}^{n_{2}} x_{3}^{n_{3}}} \\
& \times\left.\left[\frac{\partial_{4}^{n_{4}-1} \partial_{5}^{n_{5}-1} \partial_{6}^{n_{6}-1}}{\left(n_{4}-1\right) !\left(n_{5}-1\right) !\left(n_{6}-1\right) !} P\left(x_{1}, x_{2}, x_{3}+1, \ldots, x_{6}+1\right)^{D / 2-2}\right]\right|_{x_{4}, x_{5}, x_{6}=0},
\end{aligned}
$$

where we have introduced the function $\bar{t}(\underline{n}, D)$ as in general the expression given on the r.h.s. of Eq. (11) leads to a mixture of the functions $N(\underline{n}, D)$ and $t(\underline{n}, D)$. We will moreover see below that $\bar{t}(\underline{n}, D)$ obeys simple recurrence relations and in addition the extraction of $t(\underline{n}, D)$ and $N(\underline{n}, D)$ is quite simple. The resulting integral where $x_{4}=x_{5}=x_{6}=0$ is given by:

$$
\begin{aligned}
\bar{t}\left(n_{1}, n_{2}, n_{3}, D\right) & \equiv \bar{t}\left(n_{1}, n_{2}, n_{3}, 1,1,1, D\right) \\
& =\frac{1}{(2 \pi \imath)^{3}} \oint \oint \oint \frac{d x_{1} d x_{2} d x_{3}}{x_{1}^{n_{1}} x_{2}^{n_{2}} x_{3}^{n_{3}}} P\left(x_{1}, x_{2}, x_{3}+1,1,1,1\right)^{D / 2-2} \\
& =\frac{1}{(2 \pi \imath)^{3}} \oint \oint \oint \frac{d x_{1} d x_{2} d x_{3}}{x_{1}^{n_{1}} x_{2}^{n_{2}} x_{3}^{n_{3}}}\left(x_{3}^{2}-x_{1} x_{2} x_{3}+x_{1} x_{2}\left(x_{1}+x_{2}-4\right)\right)^{D / 2-2} .
\end{aligned}
$$

The function $\bar{t}\left(n_{1}, n_{2}, n_{3}, D\right)$ obeys the following recurrence relations which can be derived by using integration-by-parts in Eq. (12):

$$
\begin{aligned}
\mathbf{I}_{1}^{+} \bar{t}\left(n_{1}, n_{2}, n_{3}, D+2\right) & =(D / 2-1)\left[-\mathbf{I}_{2}^{-} \mathbf{I}_{3}^{-}+2 \mathbf{I}_{1}^{-} \mathbf{I}_{2}^{-}+\left(\mathbf{I}_{2}^{-}\right)^{2}-4 \mathbf{I}_{2}^{-}\right] \bar{t}\left(n_{1}, n_{2}, n_{3}, D\right), \\
\mathbf{I}_{2}^{+} \bar{t}\left(n_{1}, n_{2}, n_{3}, D+2\right) & =(D / 2-1)\left[-\mathbf{I}_{1}^{-} \mathbf{I}_{3}^{-}+2 \mathbf{I}_{1}^{-} \mathbf{I}_{2}^{-}+\left(\mathbf{I}_{1}^{-}\right)^{2}-4 \mathbf{I}_{1}^{-}\right] \bar{t}\left(n_{1}, n_{2}, n_{3}, D\right), \\
\mathbf{I}_{3}^{+} \bar{t}\left(n_{1}, n_{2}, n_{3}, D+2\right) & =(D / 2-1)\left[2 \mathbf{I}_{3}^{-}-\mathbf{I}_{1}^{-} \mathbf{I}_{2}^{-}\right] \bar{t}\left(n_{1}, n_{2}, n_{3}, D\right), \\
\bar{t}\left(n_{1}, n_{2}, n_{3}, D+2\right) & =\left[\left(\mathbf{I}_{3}^{-}\right)^{2}+\mathbf{I}_{1}^{-} \mathbf{I}_{2}^{-}\left(-\mathbf{I}_{3}^{-}+\mathbf{I}_{1}^{-}+\mathbf{I}_{2}^{-}-4\right)\right] \bar{t}\left(n_{1}, n_{2}, n_{3}, D\right) .
\end{aligned}
$$


The last equation immediately follows from (6).

For $n_{3}<1$ the contribution from $N(\underline{n}, D)$ vanishes as the integration around $x_{3}=0$ gives zero and only $t(\underline{n}, D)$ survives. In this case with the simple change of variables, $x_{3}=\sqrt{y_{3}}+x_{1} x_{2} / 2$, the integral (12) can be reduced to a sum over integrals which furthermore can be expressed in terms of Pochhammer symbols. Keeping in mind the normalization condition (9) we get the desired solution for $t(\underline{n}, D)$ :

$$
\begin{aligned}
t\left(n_{1}, n_{2}, n_{3}<1, D-2 c\right)= & \frac{\bar{t}\left(n_{1}, n_{2}, n_{3}, D-2 c\right)}{\bar{t}(0,0,0, D)} \\
= & \frac{(D / 2-1)_{-c}(D / 2-1 / 2)_{-c}}{(-)^{n_{3}+c} 2^{\left(2 n_{1}+2 n_{2}+6 c+3 n_{3}\right)}} \frac{(2-D)_{\left(n_{1}+n_{3}+2 c\right)}(2-D)_{\left(n_{2}+n_{3}+2 c\right)}}{(3 / 2-D / 2)_{\left(n_{1}+n_{3}+c\right)}(3 / 2-D / 2)_{\left(n_{2}+n_{3}+c\right)}} \\
& \times \sum_{k=0}^{\left[-n_{3} / 2\right]} \frac{(1 / 2)_{k}\left(n_{3}\right)_{\left(-n_{3}-2 k\right)}(D / 2-1 / 2-c)_{k}}{\left(-n_{3}-2 k\right) !\left(3 / 2-D / 2+n_{1}+n_{3}+c\right)_{k}\left(3 / 2-D / 2+n_{2}+n_{3}+c\right)_{k}} .
\end{aligned}
$$

For $n_{3}>1$ one can reduce $n_{3}$ to 1 with the help of:

$$
\begin{aligned}
\bar{t}\left(n_{1}, n_{2}, n_{3}>1, D\right) \propto \oint \oint & \oint \frac{d x_{1} d x_{2} d x_{3}}{x_{1}^{n_{1}} x_{2}^{n_{2}} x_{3}} \\
& \times\left[\frac{\partial_{3}^{n_{3}-1}}{\left(n_{3}-1\right) !}\left(x_{3}^{2}-x_{1} x_{2} x_{3}+x_{1} x_{2}\left(x_{1}+x_{2}-4\right)\right)^{D / 2-2}\right] .
\end{aligned}
$$

In connection with the reduction of $n_{3}$ two remarks are in order: First, we should mention that the reduction of $n_{3}>1$ to $n_{3}=1$ can also be expressed through a finite sum which is obvious form Eq. (18). Second, also for $n_{3}<1$ the explicit sum in Eq. (17) can be replaced by a recurrence procedure using Eqs. (15) and (16) in order to arrive at $n_{3}=0$. Afterwards $n_{1}$ and $n_{2}$ are reduced to zero using Eq. (17) with $n_{3}=0$.

For the case $n_{3}>1$ one gets after the reduction to $n_{3}=1$ a set of functions $\bar{t}\left(n_{1}, n_{2}, 1, D-\right.$ $2 c$ ) with various $n_{1}, n_{2}$ and $c$. The further reduction is done with the help of the relations:

$$
\begin{aligned}
& \bar{t}\left(n_{1}, n_{2}, 1, D\right)=\frac{(D-4)}{\left(2 n_{1}-D+2\right)}\left[\bar{t}\left(n_{1}-2, n_{2}-1,1, D-2\right)-\frac{1}{2} \bar{t}\left(n_{1}-1, n_{2}-1,0, D-2\right)\right], \\
& \bar{t}\left(n_{1}, n_{2}, 1, D\right)=\frac{\left(2 n_{2}-D+4\right)}{\left(2 n_{1}-D+2\right)} \bar{t}\left(n_{1}-1, n_{2}+1,1, D\right)+\frac{\left(n_{1}-n_{2}-1\right)}{\left(2 n_{1}-D+2\right)} \bar{t}\left(n_{1}, n_{2}+1,0, D\right),
\end{aligned}
$$

which can be obtained from Eqs. (13)-(16). Note that $\bar{t}\left(n_{1}, n_{2}, 1, D\right)=\bar{t}\left(n_{2}, n_{1}, 1, D\right)$. Therefore these equations can be used to reduce $\left(n_{1}, n_{2}\right)$ to $(0,-1),(1,0),(0,0)$ and further combinations where $n_{3}=0$. Then, using Eqs. (13) and (16) for $n_{1}, n_{2}=-1,0,1,2$, respectively, we get:

$$
\bar{t}(0,-1,1, D)=\frac{4}{3} \bar{t}(0,0,1, D)+\frac{1}{3} \bar{t}(0,0,0, D),
$$




$$
\bar{t}(1,0,1, D)=\frac{(3 D-8)}{4(D-4)} \bar{t}(0,0,1, D)-\frac{(D-2)^{2}}{8(D-3)(D-4)} \bar{t}(0,0,0, D) .
$$

Finally the shift in the last argument is fixed with the help of

$$
\begin{aligned}
\bar{t}(0,0,1, D+2) & =-\frac{2(D-2)}{3(3 D-2)(3 D-4)}[32(D-2) \bar{t}(0,0,1, D)+(11 D-16) \bar{t}(0,0,0, D)], \\
\bar{t}(0,0,0, D-2 c) & =\frac{(-)^{c}(D / 2-1)}{4^{c}(D / 2-1-c)} \frac{(D / 2-1 / 2)_{-c}}{(D / 2)_{-c}} \bar{t}(0,0,0, D),
\end{aligned}
$$

which completes the reduction of $\bar{t}\left(n_{1}, n_{2}, n_{3}, D-2 c\right)$ to $\bar{t}(0,0,1, D)$ and $\bar{t}(0,0,0, D)$. The result for $t\left(n_{1}, n_{2}, n_{3}, D-2 c\right)$ is obtained from:

$$
t\left(n_{1}, n_{2}, 1, D\right)=\frac{1}{\bar{t}(0,0,0, D)}\left[\bar{t}\left(n_{1}, n_{2}, 1, D\right)-\bar{t}(0,0,1, D) N\left(n_{1}, n_{2}, 1,1,1,1\right)\right]
$$

which follows from Eq. (91). This means that in practice from the final expression of the reduction procedure simply the coefficient of $\bar{t}(0,0,0, D)$ has to be extracted in order to get the result for $t(\underline{n}, D)$. Note that due to Eq. (17) the functions $\bar{t}\left(n_{1}, n_{2}, 0, D-2 c\right)$ in Eqs. (19)-(23) are rational in $D$, i.e., the recursion for $n_{1}, n_{2}$ and $c$ are simple oneparameter equations of the type $F(n)=a(n) F(n-1)+b(n)$ where $a(n)$ and $b(n)$ are known rational functions. Thus they can easily be solved and expressed through sums of Pochhammer symbols. At this point we refrain from listing the resulting formulas explicitly.

\section{Implementation}

In this section a possible implementation of the above formulas is discussed. We will mainly speak about the most difficult coefficient function ("T"), the others ("M" and "N") constitute simple sub-cases and can be considered in complete analogy. The calculation of the "T" coefficient function implies subsequent reduction of:

1) $n_{4}, n_{5}, n_{6}$ to 1

2) $n_{3}$ to 0 or 1 ,

3) $n_{1}, n_{2}$ to $(0,0),(1,0),(0,-1)$,

4) $D-2 c$ to $D$.

The steps 1) and 2) demand a Taylor expansion of a polynomial raised to non-integer power. This can either be done by direct differentiation (see Eqs. (11) and (18)), or by computing multiple combinatoric sums which arise after rewriting the action of the operators $\partial^{n} / \partial x_{i}^{n}$. Moreover, the reduction of each parameter can be done independently by one of these methods. The steps 3) and 4) can be performed either by using the recursion relations (19)-(24) or by explicitly evaluating the sums. 
In practice, the quantities of physical interest can be expressed as linear combination of vacuum integrals, the number of which can be very large (up to $10^{7}$; see, e.g. [2]). It is very natural to perform the reductions 1)-4) not for individual integrals, but for the whole expression as simplifications may take place in intermediate steps of the calculation. Of course, the method which gives the best performance strongly depends on the computer algebra system used. In the following we compare REDUCE 3.6 and FORM 2.3 both running on a DEC-Alpha-station with $266 \mathrm{MHz}$.

REDUCE. Our experience shows that the method using differentiation in steps 1) and 2 ) works about two times faster, although the calculation of the multiple sums allows to save computer memory.

At step 3) the recurrence procedure is also about two times faster than the use of explicit sums. The higher demand concerning the memory is not essential at this point as for step 1) more memory is needed and thus the limitations are given from there. Step 4) needs negligible time and memory. Here in practice the recursion approach is used.

FORM. The explicit differentiation using FORM requires for the variables to be defined as "non-commuting" 11]. This has the consequence that the sorting of the intermediate expressions becomes quite slow and needs in addition more main memory. Therefore in practice only the method relying on multiple sums are effective for step 1). At steps 2) and 3 ) we can choose between the combinatoric-sum and the recursion approach. It turned out that their performance is comparable. At step 4) we prefer to use a one-parameter table for the functions $t(0,0,1, D-2 c)$.

The implementation in FORM is done in such a way that an expansion in $D-4$ is performed for the intermediate expressions. Thereby some tricks were used like the application of the "ACCU" function which collects polynomials in $D-4$ or the use of tables for the Pochhammer symbols [11]. Note that explicit formulas are used for the realization. Therefore one has full control on the powers of intermediate poles in $D-4$. This actually is a problem in the approach based on recurrence relations only. There the expansion in $D-4$ has to be performed to higher order which has to be fixed after careful examination of the recurrence relations.

It is interesting to mention that the implementation within REDUCE, which even computes the full $D$-dependence, is about three times faster than the one using FORM. An explanation for this unexpected fact is the possibility to use direct differentiation within REDUCE. It might also be possible that during the calculation essential cancellations among long sums containing Pochhammer symbols take place. REDUCE with its ability to work with rationals can exploit this fact, however, FORM cannot. We should also mention that the attempt to expand in $D-4$ in the "REDUCE" version (using "wtlevel" declaration [10]) takes more time but is still faster than FORM. 
Within FORM we are also in the position to compare the recursion approach [3, [4] where, however, in addition to the recurrence relations also tables were used [12], with the one described in this paper. As an example we considered the contribution of the non-planar diagram to the photon propagator and performed an expansion up to $\mathcal{O}\left(q^{12}\right)$ where $q$ is the external momentum. It turned out, that the "improved" recursion works better for planar vacuum integrals contributing to this diagram, however, worse for the non-planar ones. The joined variant which uses "improved" recursion for the planar diagrams and the new method for non-planar ones gives a gain of roughly $8 \%$ as compared to the use of only the "improved" recursion.

\section{Conclusions}

The new approach described in this paper allows to solve the recurrence relations for three-loop vacuum integrals explicitly and expresses the result in terms of multiple sums containing Pochhammer symbols. Nevertheless, the final choice of the algorithm which leads to the best performance strongly depends on the peculiarities of the problem and also on the symbolic language used.

For the particular problem considered here - three-loop integrals with four massive and two massless lines - we get better results using REDUCE 3.6 than using FORM 2.3 from point of view of CPU time and in the presence of sufficient memory resources. Certainly, FORM stands beyond the competition as soon as the intermediate expressions become very large and enormous swapping to the hard disk is necessary.

\section{Acknowledgment}

We would like to thank J.H. Kühn and K.G. Chetyrkin for stimulating discussions. P.A.B. thanks the University of Karlsruhe for the hospitality during the visit when an important part of this work has been done.

\section{References}

[1] P. A. Baikov and D. J. Broadhurst, Presented at 4th International Workshop on Software Engineering and Artificial Intelligence for High Energy and Nuclear Physics (AIHENP95), Pisa, Italy, 3-8 April 1995. Published in Pisa AIHENP (1995) 167.

[2] K. G. Chetyrkin, J. H. Kühn and M. Steinhauser, Phys. Lett. B371 (1996) 93 Nucl. Phys. B482 (1996) 213; Nucl. Phys. B505 (1997) 40. 
[3] K. G. Chetyrkin and F. V. Tkachov, Nucl. Phys. B192 (1981) 159;

F. V. Tkachov, Phys. Lett. B100 (1981) 65.

[4] D. J. Broadhurst, Z. Phys. C54 (1992) 559.

[5] O. V. Tarasov, Nucl. Phys. B502 (1997) 455.

[6] L. V. Avdeev, Comput. Phys. Commun. 98 (1996) 15.

[7] F. V. Tkachov, Theor. Mat. Fiz. 56 (1983) 350.

[8] S. A. Larin, F. V. Tkachev and J. A. M. Vermaseren, NIKHEF Report No. NIKHEF-H/91-18 (September 1991).

[9] P. A. Baikov, Phys. Lett. B385, (1996) 404; Nucl. Instr. \& Methods A389 (1997) 347.

[10] A. C. Hearn, REDUCE User's Manual, version 3.6.

[11] J. A. M. Vermaseren, Symbolic Manipulation with FORM, version 2.

[12] M. Steinhauser, Ph.D. thesis, Karlsruhe University (Shaker Verlag, Aachen, 1996). 